\title{
Gelsemine and Gelsemium sempervirens L. extracts in animal behavioral test: comments and related biases
}

\section{Salvatore Chirumbolo*}

Department of Pathology and Diagnostics, University of Verona, Verona, Italy

*Correspondence: salvatore.chirumbolo@univr.it

\section{A commentary on}

Dose-effect study of Gelsemium sempervirens in high dilutions on anxiety-related responses in mice

by Magnani, P., Conforti, A., Zanolin, E., Marzotto, M., and Bellavite, P. (2010). Psychopharmacology (Berl.) 210, 533-545.

Recently reported papers have shown the role of the alkaloid gelsemine in behavioral science (Dutt et al., 2010). Other issues have yet raised questions about cytotoxicity of Gelsemium compounds: plants belonging to the genus Gelsemium are highly poisonous but toxicological evaluation of suspected poisoning cases has been hampered by the chemical complexity of the Gelsemium toxins involved (Lai and Chan, 2009). Gelsemium extracts are, in fact, potentially genotoxic. Evidence was reported showing that the $95 \%$ ethanol extract of Gelsemium sempervirens L. plays an inhibitory activity against human DNA topoisomerase I (Zhang et al., 2008). Phytochemical investigations of this active extract resulted in the isolation and identification of several steroids, in addition to scopoletin and its derivatives, gelsemine, sempervirine, uvaol, and 2-(4-hydroxyphenyl)-ethyl heptadecanoate (Zhang et al., 2008). Actually, cytotoxic steroids of Gelsemium plant were described elsewhere in the past (Schun and Cordell, 1987). G. sempervirens (L.) (Loganiaceae) has been recommended for a long time for the relief of anxiety in traditional folk medicines, although no pharmacological studies evaluated it in this regard, prior to the recently reported evidence of Dutt et al. (2010). The effect of this medicinal plant has been investigated also in complementary, traditional medicine, and ethnopharmacology, with good results in vitro, as very low doses of Gelsemium extracts has shown to reduce anxiety in animal models (Magnani et al., 2010), an evidence that might suggest the possibility for a reduction of side effects due to Gelsemium noxious compounds if more selective compounds can be identified.

Centesimal (C) dilutions of G. sempervirens $(4 \mathrm{C}, 5 \mathrm{C}, 7 \mathrm{C}, 9 \mathrm{C}$, and $30 \mathrm{C})$ appeared able to change significantly mouse behavioral effects as assessed in the open-field (OF) and light-dark (LD) tests in blind and randomized fashion (Magnani et al., 2010) but effects in the elevated plus-maze, an assay considered to be important in behavioral assessment of potential anxiolytic compounds (Ramos, 2008), have not been assessed.

Actually, the physical integration of different current tests in one single apparatus, in such a way that the emotional status of an animal becomes assessable through a series of distinct tasks, should contribute to increased reliability, rapidity, and comprehensiveness in behavioral testing. Although manuscripts in the literature have reported showing an anxiolytic effect of $G$. sempervirens at concentrations lower than $1.67 \mathrm{ymol} / \mathrm{L}$, criticism may be raised about biases in their experimental context (Venard et al., 2009; Magnani et al., 2010). When a preparation according to the homeopathic pharmacopeia is assayed in a behavioral experimental setting with animals, the contribution of biases may overwhelm any reliable output (Bellavite et al., 2009). The main active substance in G. sempervirens causing an anxiolytic action is believed to be gelsemine (Bellavite, et al.,2009). Gelsemine, is an alkaloid that contains the same functional groups as strychnine and has been shown to increase $3 \alpha, 5 \alpha$-tetrahydroprogesterone or allopregnenolone ( $3 \alpha, 5 \alpha$-THP) neurosteroid synthesis (Venard et al., 2008). The stimulatory effects of glycine and gelsemine on $3 \alpha, 5 \alpha$-THP production were additive when the two drugs were combined. These results may suggest that glycine and gelsemine, acting via glycine receptor (Gly$\mathrm{R}$ ), upregulate $3 \alpha, 5 \alpha$-THP biosynthesis in the spinal cord (Venard et al., 2008). Some pilot data have also raised the possibility that chronically administered neurosteroids antagonize certain acute effects of benzodiazepines and may enhance arousal via antagonist or inverse agonist actions at the benzodiazepine/GABA(A) receptor complex (Venard et al., 2008). As several complex issues were raised, the use of Gelsemium in behavioral science should undergo some reappraisal.

In this field, a possible main critical topic might be the placebo/nocebo response of animals, able to exert biases on anxiety models. In medicine, the placebo response or placebo effect has often been regarded as a nuisance in basic research and particularly in clinical research, but very few works were related to animal behavior (McMillan, 1999). The latest scientific evidence has suggested, however, that the placebo effect and the nocebo effect, the negative effects of placebo, stem from highly active processes in the brain that are mediated by psychological mechanisms such as expectation and conditioning (Enck et al., 2008). Pavlovian conditioning might justify a placebo effect in housed laboratory animals (McDannald, 2010). Animals which undergo the same stressing procedure for several times (such as intra-peritoneal injections) may relate the physical stressor to other stimuli, such as operator's odor or voice-sound, particularly when the same operator performs the injections. This bias is not always considered, although wash out and blinding randomized protocols are followed (Chirumbolo, 2011). The search for biases in animal behavior research should be a hallmark of any published paper on the topic. Some simple biases, for example animal sex (as researchers routinely use only male animals), have raised criticism concerning the opportunity to use these models to test drugs that would be used by the human female population (Wald and $\mathrm{Wu}$, 2010). Placebo/nocebo response, as an effect of a conditioning/learning mechanism, is almost never considered in behavioral testing with laboratory animals: actually, none of the recently published papers about the 
anxiolytic activity of $G$. sempervirens has referred to the possibility of the placebo effect (McMillan, 1999; Lewejohann et al., 2006; Enck et al.,2008; Colloca et al., 2010). Pavlovian fear conditioning (PFC) is a typical behavioral paradigm in which animals learn to predict aversive events: an aversive stimulus (e.g., a sting) is associated with a particular neutral context (e.g., a tone or a smell), resulting in the expression of fear responses to the originally neutral stimulus or context (Maren, 2001). This phenomenon depends on environmental features, so even the room or the time in a day in which PFC is elicited may constitute another bias in behavioral tests (Magnani et al., 2010). The same mechanism could elicit in animals a placebo response. This bias may affect statistical evaluation of outcoming results (Nakagawa, 2004).

When plant derivatives are used in behavioral sciences, many factors contribute to make rather tricky the interpretation of the data. For example, intrinsic biases in the statistical computation are often hidden. According to some Authors, the statistical power of behavioral studies to detect relationships is quite low: the power to detect a medium effect is $39-47 \%$ and in this case only $10-20 \%$ of tests exceeded the recommended minimum criterion of $80 \%$, as assessed by Cohen and colleagues (Cohen, 1988; Jennions and Møller, 2003). Biases can be unknowingly brought in by researchers themselves: they may be disinclined to increase sample sizes when they infer that there is no significant effect to detect and this would also yield a negative correlation between power and $p$ value (Jennions and Møller, 2003). This is a rational, but worrying, behavior because studies with significant results are more likely to be published than those without: this is particularly true in high dilution or homeopathic research (Bonamin and Endler, 2010). Hence, in behavioral tests, not only a randomized double blinding approach and a prevention of PFC mechanism but also a biases analysis should be performed in order to assess the reliability of experimental results (Greenland, 1996). Recent neuroimaging studies of conditioned fear, attention to threat and interpretation of emotionally ambiguous stimuli indicate common amygdala-prefrontal circuitry underlying these processes, and suggest that the balance of activity within this circuitry is altered in anxiety, creating a bias toward threat-related responses. This provides a focus for future translational research, and targeted pharmacological and cognitive interventions (Bishop, 2007). Besides randomized blinding procedures, many researchers treat animal models with the same experimental standardization and evaluation applied to cells; notwithstanding, animals produce effects in response to external stimuli that are not so homogenously related to an environmental background. Environmental factors play their significant role. With regard to the differences between enriched and unstructured housing conditions, overall consistent results were achieved by different experimenters in both laboratories; the reliability of behavioral phenotyping is not challenged seriously by experimenter and laboratory environment as long as appropriate standardizations are met and suitable controls are involved (Lewejohann et al., 2006). Certainly, research on the antianxiety potential of Gelsemium alkaloids needs further investigative efforts, in order to comprehend the role of this plant in neuropharmacology.

\section{REFERENCES}

Bellavite, P., Magnani, P., Marzotto, M., and Conforti, A. (2009). Assays of homeopathic remedies in rodent behavioural and psychopathological models. Homeopathy 98, 208-227.

Bishop, S. J. (2007). Neurocognitive mechanisms of anxiety: an integrative account. Trends Cogn. Sci. 11, 307-316.

Bonamin, L. V., and Endler, P. C. (2010). Animal models for studying homeopathy and high dilutions: conceptual critical review. Homeopathy 99, 37-50. [Erratum in: Homeopathy 2010, 99, 152].

Chirumbolo, S. (2011). Randomized blinding in basic research of homeopathy: some comments. Complement. Ther. Med. 19,107-108.

Cohen, J. (1988). Statistical Power Analysis for the Behavioural Sciences, 2nd Edn, Hillsdale, NJ: Lawrence Erlbaum.

Colloca, L., Petrovic, P., Wager, T. D., Ingvar, M., and Benedetti, F. (2010). How the number of learning trials affects placebo and nocebo responses. Pain 151, 430-439.

Dutt, V., Dhar, V. J., and Sharma, A. (2010). Antianxiety activity of Gelsemium sempervirens. Pharm. Biol. 48, 1091-1096.

Enck, P., Benedetti, F., and Schedlowski, M. (2008). New insights into the placebo and nocebo responses. Neuron 59, 195-206.
Greenland, S. (1996). Basic methods for sensitivity analysis of biases. Int. J. Epidemiol. 25, 1107-1116.

Jennions, M. D., and Møller, A. P. (2003). A survey of the statistical power of research in behavioural ecology and animal behavior. Behav. Ecol. 14, 438-445.

Lai, C. K., and Chan, Y. W. (2009). Confirmation of Gelsemium poisoning by targeted analysis of toxic Gelsemium alkaloids in urine. J. Anal. Toxicol. 33, 56-61.

Lewejohann, L., Reinhard, C., Schrewe, A., Brandewiede, J., Haemisch,A., Görtz, N., Schachner,M., and Sachser, N (2006). Environmental bias? Effects of housing conditions, laboratory environment and experimenter on behavioral tests. Genes Brain Behav. 5, 64-72.

Magnani, P., Conforti, A., Zanolin, E., Marzotto, M., and Bellavite, P. (2010). Dose-effect study of Gelsemium sempervirens in high dilutions on anxiety-related responses in mice. Psychopharmacology (Berl.) 210, 533-545.

Maren, S. (2001). Neurobiology of Pavlovian fear conditioning. Annu. Rev. Neurosci. 24, 897-931.

McDannald, M.A. (2010). Contributions of the amygdala central nucleus and ventrolateral periaqueductal grey to freezing and instrumental suppression in Pavlovian fear conditioning. Behav. Brain Res. 211, 111-117.

McMillan, F. D. (1999). The placebo effect in animals. J. Am. Vet. Med. Assoc. 215, 992-999.

Nakagawa, S. (2004). A farewell to Bonferroni: the problems of low statistical power and publication bias. Behav. Ecol. 15, 1044-1045.

Ramos, A. (2008). Animals models of anxiety: do I need multiple tests? Trends Pharmacol. Sci. 29, 493-498.

Schun, Y., and Cordell, G. A. (1987). Cytotoxic steroids of Gelsemium sempervirens. J. Nat. Prod. 50, 195-198.

Venard, C., Boujedaini, N., Belon, P., Mensah-Nyagan, A. G., and Patte-Mensah, C. (2008). Regulation of neurosteroid allopregnanolone biosynthesis in the rat spinal cord by glycine and the alkaloidal analogs strychnine and gelsemine. Neuroscience 153, 154-181.

Venard, C., Boujedaini, N., Mensah-Nyagan, A. G., and Patte-Mensah, C. (2009). Comparative analysis of gelsemine and Gelsemium sempervirens activity on neurosteroid allopregnanolone formation in the spinal cord and limbic system. Evid. Based Complement. Alternat. Med. 5, 80-89.

Wald, C., and Wu, C. (2010). Of mice and women: the bias in animal models. Science 327, 1571-1572.

Zhang, Z., Wang, P., Yuan, W., and Li, S. (2008). Steroids, alkaloids and coumarins from Gelsemium sempervirens. Planta Med. 74, 1818-1822.

Received: 28 February 2011; accepted: 05 May 2011; published online: 16 May 2011.

Citation: Chirumbolo S (2011) Gelsemine and Gelsemium sempervirens $L$. extracts in animal behavioral test: comments and related biases. Front. Neur. 2:31. doi: 10.3389/ fneur.2011.00031

This article was submitted to Frontiers in Neuropharmacology, a specialty of Frontiers in Neurology.

Copyright (c) 2011 Chirumbolo. This is an open-access article subject to a non-exclusive license between the authors and Frontiers Media SA, which permits use, distribution and reproduction in other forums, provided the original authors and source are credited and other Frontiers conditions are complied with. 